\title{
Suboptimal Model Predictive Control of Hybrid Systems Based on Mode-Switching Constraints
}

\author{
A. Ingimundarson, C. Ocampo-Martinez and A. Bemporad
}

\begin{abstract}
Model predictive control (MPC) is recognized as a very versatile and effective way of controlling constrained hybrid dynamical systems in closed-loop. The main drawback of hybrid MPC is the heavy computation burden of the associated on-line mixed-integer optimization. Explicit MPC solutions overcome such a problem by rewriting the control law in piecewise affine form, but are limited to relatively simple hybrid control problem setups. This paper presents an alternative approach for reducing the complexity of computations by suitably constraining the mode sequence over the prediction horizon, so that on-line optimization is solved more quickly. While tracking performance of the feedback loop may be affected because of the suboptimality of the approach, closedloop stability is guaranteed. The effectiveness of the method is demonstrated by an example.
\end{abstract}

Index Terms-Hybrid systems, model predictive control, mixed integer programming, large scale systems

\section{INTRODUCTION}

An important issue related to on-line implementation of hybrid Model Predictive Control (MPC) [1] is the time required for the on-line computation of the associated Mixed Integer linear or quadratic Program (MIP). Being MIP $\mathcal{N} \mathcal{P}$ hard, the worst case computation time grows exponentially with the number of integer variables that describe the evolution of the hybrid model over the prediction horizon. Despite the advance in presolving techniques and the efficiency of MIP solvers in taking advantage of infeasibility of certain nodes in the search tree, especially through the use of efficient cuts added during the search, still the complexity of Mixed Integer Quadratic Programs (MIQP) or Mixed Integer Linear Programs (MILP) may be prohibitive in applications of hybrid MPC of relatively large-scale systems.

Even if the average computation time for a particular hybrid MPC problem is satisfactory, as the number of infeasible nodes may largely change with the current state of the plant during the execution of the control algorithm, the worst case computation time per sampling step of the MIP is often unpredictable.

To circumvent such unpredictability and therefore to be able to enforce hard real-time computation constraints, in this article a hybrid MPC strategy is proposed which limits the number of nodes that are feasible in the MIP problem on-line. This is done by adding constraints to the MIP based

A. Ingimundarson and C. Ocampo-Martinez are with the Automatic Control Department, Technical University of Catalonia (UPC), Rambla Sant Nebridi, 10, 08222 Terrassa, Spain. <ari.ingimundarson, carlos.ocampo>@upc.edu

A. Bemporad is with the Department of Information Engineering, University of Siena, Via Roma 56, I-53100 Siena, Italy. bemporad@dii.unisi.it on insight into the system dynamics, therefore helping the MIP solver by adding cuts in the search space. In this way the main source of complexity, namely the combinatorial explosion related to the binary search tree, is arbitrarily limited, at the expense of a suboptimal solution. The strategy is easy to implement as it only involves adding linear inequalities to the MIP problem at hand.

The strategy proposed is suboptimal but stability is proved using recent stability results for hybrid MPC [2]. It has been recognized in the MPC literature that even though the solution applied is only suboptimal, stability can often be proven [3].

The strategy presented was motivated by hybrid MPC of sewer networks where hundreds of binary variables are necessary to describe the evolution of the system over the prediction horizon [4]. In the sewer network case, many modes can only be reached when the system is excited by large disturbances (heavy rain). An example would be overflow in catchments in certain parts of a city.

This article treats receding horizon control (RHC) of discrete-time hybrid dynamical systems in Mixed Logical Dynamical (MLD) form [1]. Other RHC approaches for hybrid systems are [5], [6]. Optimal control of hybrid systems has been treated also in [7], [8], [9], [10].

This manuscript is organized as follows. In Sections II and III the problem is introduced and a proof of stability of the proposed scheme is given. Practical issues of the proposed method are discussed in IV. In order to show the application of the proposed approach, in Section $\mathrm{V}$ an illustrative example is given and finally conclusions are drawn in Section VI.

\section{PRELIMINARIES}

Consider the Mixed Logical Dynamical (MLD) hybrid model

$$
\begin{aligned}
& x_{k+1}=A x_{k}+B_{1} u_{k}+B_{2} \delta_{k}+B_{3} z_{k} \\
& E_{2} \delta_{k}+E_{3} z_{k} \leq E_{1} u_{k}+E_{4} x_{k}+E_{5}
\end{aligned}
$$

where $x_{k} \in \mathbb{X} \subseteq \mathbb{R}^{n}$ is the state vector and $u_{k} \in \mathbb{U} \subseteq \mathbb{R}^{m}$ is the vector of manipulated variables (inputs). $\mathbb{X}$ and $\mathbb{U}$ are polyhedra containing the origin in their interior, and may include state and input constraints, respectively, that need to be enforced by the MPC control design. The binary vector $\delta_{k}=\left[\delta_{k}^{1}, \ldots, \delta_{k}^{r_{l}}\right] \in\{0,1\}^{r_{l}}$ of dimension $r_{l}$ and the continuous-valued vector $z_{k} \in \mathbb{R}^{r_{c}}$ of dimension $r_{c}$ are the vectors of auxiliary variables associated with the MLD form. A specific value of the variable $\delta_{k}$ is referred to as a mode of the hybrid system. Equation (1) collects the set of 
constraints on system variables as well as translations from logic propositions [11].

\section{Notation}

In the sequel, we denote by $\mathbb{Z}, \mathbb{Z}_{+}$the set of integer numbers and the set of non-negative integer numbers, respectively. In the same manner, $\mathbb{R}$ and $\mathbb{R}_{+}$denote the set of real numbers and the set of non-negative real numbers, respectively. The notation $\mathbb{Z}_{\geq c}$ denotes the set $\{k \in \mathbb{Z} \mid k \geq c\}$ for some $c \in \mathbb{Z}$. int $(\mathbb{Y})$ denotes the interior of the set $\mathbb{Y}$ and $\mathbb{Y}^{N} \triangleq \mathbb{Y} \times \mathbb{Y} \times \cdots \times \mathbb{Y}$. For a vector $q \in \mathbb{R}^{n}$, the expression $\|q\|_{p}$ denotes an arbitrary Hölder vector $p$-norm, defined for $p$ on the interval $1 \leq p \leq \infty$.

\section{Hybrid MPC PRoblem WITH CONSTRAINTS ON THE Mode SeQuence}

This section explains the details of the proposed suboptimal approach and the conditions for feasibility and closedloop stability. Some definitions and results of this section follow closely Sections II and III of [2]. Assume that there are no disturbances. The mapping of state $x_{k}$ and control signal $u_{k}$ defined by the MLD equation (1) is denoted as:

$$
x_{k+1}=g\left(x_{k}, u_{k}\right)
$$

where $g: \mathbb{R}^{n} \times \mathbb{R}^{m} \rightarrow \mathbb{R}^{n}$ is the state update function (usually discontinuous, because of the binary nature of $\delta_{k}$ ) defined by (1) and $k \in \mathbb{Z}_{+}$. It is assumed that the origin is an equilibrium state with $u_{k}=0$, i.e., $g(0,0)=0$.

For a fixed prediction horizon $N \in \mathbb{Z}_{\geq 1}$, let $X_{k}\left(x_{k}, U_{k}\right) \triangleq$ $\left(x_{1 \mid k}, x_{2 \mid k} \ldots x_{N \mid k}\right) \in \mathbb{X}^{N}$ denote the state sequence generated by the MLD system (2) from initial state $x_{0 \mid k} \triangleq x_{k}$ and by applying the input sequence $U_{k} \triangleq\left(u_{0 \mid k}, \ldots, u_{N-1 \mid k}\right) \in$ $\mathbb{U}^{N}$. Related to these sequences is the mode sequence $\Delta_{k}\left(x_{k}, U_{k}\right)=\left(\delta_{0 \mid k}, \ldots, \delta_{N-1 \mid k}\right) \in\{0,1\}^{r_{l} \times N}$ of binary vectors $\delta_{k}$ uniquily defined by (1b) when $U_{k}$ is applied to (1) from initial state $x_{k}$. Let $\mathbb{X}_{T}$ denote a target state set that contains the origin in its interior.

Let $\bar{\Delta}_{k}=\left(\bar{\delta}_{0 \mid k}, \ldots, \bar{\delta}_{N-1 \mid k}\right) \in\{0,1\}^{r_{l} \times N}$ be a reference sequence of binary variables $\bar{\delta}_{k}$ of the same dimension as $\Delta_{k}$ and define the sets $\mathcal{D}_{M_{i}}\left(\bar{\Delta}_{k}\right) \subseteq\{0,1\}^{r_{l} \times N}$ and $\mathcal{D}_{M}\left(\bar{\Delta}_{k}\right) \subseteq$ $\{0,1\}^{r_{l} \times N}$ in the following manner:

$$
\begin{aligned}
& \mathcal{D}_{M_{i}}\left(\bar{\Delta}_{k}\right)=\left\{\Delta_{k} \in\{0,1\}^{r_{l} \times N}\left|\sum_{k=0}^{N-1}\right| \bar{\delta}_{k}^{i}-\delta_{k}^{i} \mid \leq M_{i}\right\} \\
& \mathcal{D}_{M}\left(\bar{\Delta}_{k}\right)=\left\{\Delta_{k} \in\{0,1\}^{r_{l} \times N}\left|\sum_{i=1}^{r_{l}} \sum_{k=0}^{N-1}\right| \bar{\delta}_{k}^{i}-\delta_{k}^{i} \mid \leq M\right\} \text { (4) }
\end{aligned}
$$

where $M, M_{i} \in \mathbb{Z}_{+}$are given bounds on the number of switches from the reference sequence, and $i=1 \ldots r_{l}$. The dependence of $\Delta_{k}$ on $x_{k}$ and $U_{k}$ is omitted for compactness of notation.

The sets in (3), (4) contain all sequences $\Delta_{k}$ having a limited number of differences from the reference sequence $\bar{\Delta}_{k}$. Thinking of $\Delta_{k}$ and $\bar{\Delta}_{k}$ as binary strings, the inequalities in (3), (4) limit the Hamming distance between such strings. In what follows, the discussion will be limited to the set $\mathcal{D}_{M}\left(\bar{\Delta}_{k}\right)$ for brevity, although the results can be easily extended to $\mathcal{D}_{M_{i}}\left(\bar{\Delta}_{k}\right)$.

The class of admissible input sequences defined with respect to $\mathbb{X}_{T}$ and set $\mathcal{D}_{M}$ is:

$$
\begin{aligned}
\mathcal{U}_{N}\left(x_{k}, \bar{\Delta}_{k}\right) \triangleq & \left\{U_{k} \in \mathbb{U}^{N} \mid X_{k}\left(x_{k}, U_{k}\right) \in \mathbb{X}^{N},\right. \\
& \left.x_{N \mid k} \in \mathbb{X}_{T}, \Delta_{k}\left(x_{k}, U_{k}\right) \in \mathcal{D}_{M}\left(\bar{\Delta}_{k}\right)\right\}
\end{aligned}
$$

Remark 1: Notice that the set $\mathcal{U}_{N}\left(x_{k}, \bar{\Delta}_{k}\right)$ can be characterized exactly through a set of mixed integer linear inequalities. [2].

The MPC-problem will be stated in a similar way as in

Problem 1 (MPC-problem with Mode Constraints): Let the target set $\mathbb{X}_{T} \subset \mathbb{X}$ and $N \in \mathbb{Z}_{+}$be given. Minimize the cost function

$$
J\left(x_{k}, U_{k}\right)=F\left(x_{N \mid k}\right)+\sum_{i=0}^{N-1} L\left(x_{i \mid k}, u_{i \mid k}\right)
$$

over $U_{k} \in \mathcal{U}_{N}\left(x_{k}, \bar{\Delta}_{k}\right)$ where $F: \mathbb{R}^{n} \rightarrow \mathbb{R}_{+}$and $L: \mathbb{R}^{n} \times$ $\mathbb{R}^{m} \rightarrow \mathbb{R}_{+}$are functions that fulfill $F(0)=0$ and $L(0,0)=$ 0.

An initial state $x_{0} \in \mathbb{X}$ is feasible if there exist a reference sequence $\bar{\Delta}_{k}$ such that $\mathcal{U}_{N}\left(x_{0}, \bar{\Delta}_{k}\right) \neq \emptyset$. Let the set $\mathbb{X}_{f}(N) \subseteq \mathbb{X}$ denote the set of feasible states. The function

$$
V_{\mathrm{MPC}}\left(x_{k}\right)=\min _{U_{k} \in \mathcal{U}_{N}\left(x_{k}, \bar{\Delta}_{k}\right)} J\left(x_{k}, U_{k}\right)
$$

related to Problem 1 is called the MPC value function. It is assumed that at all time instants $k \in \mathbb{Z}_{+}$the state $x_{k} \in \mathbb{X}_{f}(N)$, i.e., there exists a corresponding optimal control sequence

$$
U_{k}^{*}=\left(u_{0 \mid k}^{*}, u_{1 \mid k}^{*}, \ldots, u_{N-1 \mid k}^{*}\right) .
$$

Using the receding horizon philosophy [12], [13], the MPC control law is defined as:

$$
u_{\mathrm{MPC}}\left(x_{k}\right) \triangleq u_{0 \mid k}^{*}
$$

where $u_{0 \mid k}^{*}$ is the first element of $U_{k}^{*}$.

According to [2], both feasibility and stability can be ensured by using a terminal cost and constraint set method as in [3] but with the conditions and assumptions adapted to hybrid systems. Therefore, the following assumption from [2] is now presented to prove stability of the closed-loop system (2) and (9).

Assumption 1 (see [2]): Assume there exist strictly increasing, continuous functions $\alpha_{1}, \alpha_{2}: \mathbb{R}_{+} \rightarrow \mathbb{R}_{+}$that fulfill $\alpha_{1}(0)=\alpha_{2}(0)=0$, a neighborhood of the origin $\mathcal{N} \subset$ $\mathbb{X}_{f}(N)$ and a nonlinear, possibly discontinuous function $h: \mathbb{R}^{n} \rightarrow \mathbb{R}^{m}$, such that $\mathbb{X}_{T} \subset \mathbb{X}_{\mathbb{U}}$, with $0 \in \operatorname{int}\left(\mathbb{X}_{T}\right)$, is a positively invariant set for system (2) in closed loop with $u_{k}=h\left(x_{k}\right) . \mathbb{X}_{\mathbb{U}}$ denotes the safe set with respect to state and input constraints for $h(\cdot)$. Furthermore,

$$
\begin{array}{rc}
L(x, u) & \leq \alpha_{1}(\|x\|), \forall x \in \mathbb{X}_{f}(N), \forall u \in \mathbb{U},(10) \\
F(x) & \geq \alpha_{2}(\|x\|), \forall x \in \mathcal{N} \text { and } \\
F(g(x, h(x)) & -F(x)+L(x, h(x)) \leq 0, \forall x \in \mathbb{X}_{T} .
\end{array}
$$


Remark 2: The selection of the reference sequence of logical variables between samples $k$ and $k+1$ can be done using the control law $u_{k}=h\left(x_{k}\right)$ and considering the sequence $\Delta_{k}^{*}=\left(\delta_{0 \mid k}^{*}, \delta_{1 \mid k}^{*}, \ldots, \delta_{N-1 \mid k}^{*}\right)$ obtained from the solution of Problem 1 at time $k$. Given $\vartheta=h\left(x_{N \mid k}^{*}\right)$ the reference sequence of logical variables in time $k+1$ is put as:

$$
\bar{\Delta}_{k+1}=\left(\delta_{1 \mid k}^{*}, \ldots, \delta_{N-1 \mid k}^{*}, \delta_{+}\left(x_{N \mid k}^{*}, \vartheta\right)\right)
$$

where $\delta_{+}\left(x_{N \mid k}^{*}, \vartheta\right)$ is determined through equation (1b).

The following theorem is now presented for stability of MPC controllers with constraints on the mode sequence. Its proof follows closely the proof presented in [2]. It rests on Lyapunov stability results for systems with discontinuous system dynamics developed in [14] but it also takes into account the introduced mode-sequence constraints.

Theorem 1: Given a prediction horizon $N$, suppose that Assumption 1 holds. Then it holds that:

1) If Problem 1 is feasible at time $k$ for state $x_{k} \in \mathbb{X}$, then it is also feasible at time $k+1$ for state $x_{k+1}=$ $g\left(x_{k}, u_{\mathrm{MPC}}\left(x_{k}\right)\right)$ and $\mathbb{X}_{T} \subseteq \mathbb{X}_{f}(N)$.

2) $\mathbb{X}_{T} \subset \mathbb{X}_{f}(N)$;

3) The origin of the MPC closed-loop system formed by applying control law (9) to plant (2) is asymptotically stable in the Lyapunov sense for initial conditions in $\mathbb{X}_{f}(N)$.

Proof:

Consider the shifted sequence

$$
U_{k+1}^{1} \triangleq\left(u_{1 \mid k}^{*}, \ldots, u_{N-1 \mid k}^{*}, h\left(x_{N-1 \mid k+1}\right)\right)
$$

where $x_{N-1 \mid k+1}$ is the state at prediction time $N-$ 1 , obtained at time $k+1$ by applying the input sequence $u_{1 \mid k}^{*}, \ldots, u_{N-1 \mid k}^{*}$ to system (2) with initial condition $x_{0 \mid k+1} \triangleq x_{1 \mid k}^{*}=x_{k+1}=g\left(x_{k}, u_{\mathrm{MPC}}\left(x_{k}\right)\right)$. Note that $x_{N-1 \mid k+1}=x_{N \mid k}^{*}$.

(1): If Problem 1 is feasible at time $k \in \mathbb{Z}_{+}$for state $x_{k} \in \mathbb{X}$ then there exists a reference sequence $\bar{\Delta}_{k}$ such that $\mathcal{U}\left(x_{k}, \bar{\Delta}_{k}\right)$ is nonempty. The optimal solution to Problem 1 is denoted by $U_{k}^{*}$. Then it follows that $x_{N-1 \mid k+1} \in \mathbb{X}_{T}$. Due to Remark 2 and the positive invariance of $\mathbb{X}_{T} \subseteq \mathbb{X}_{\mathbb{U}}$, it holds that $x_{N \mid k+1} \in \mathbb{X}_{T}$ and $U_{k+1}^{1} \in \mathcal{U}\left(x_{k+1}, \bar{\Delta}_{k+1}\right)$. This implies that Problem 1 is feasible at time $k+1$ for $x_{k+1}$ and binary reference sequence $\bar{\Delta}_{k+1}$.

(2): Let $\tilde{X}\left(x_{k}\right)=\left(\tilde{x}_{1 \mid k}, \tilde{x}_{2 \mid k} \ldots \tilde{x}_{N \mid k}\right)$ denote the state sequence generated by the system $x_{k+1}=g\left(x_{k}, h\left(x_{k}\right)\right)$ from initial state $\tilde{x}_{0 \mid k}=x_{k} \in \mathbb{X}_{T}$. Let $\tilde{U}_{k}$ denote the corresponding control signal. Since $\tilde{X}_{k} \in \mathbb{X}_{T}^{N}$, then $\tilde{U}_{k} \in \mathbb{U}$ according to Assumption 1. A candidate reference sequence $\bar{\Delta}_{k}$ so that $\mathcal{U}_{k}\left(x_{k}, \bar{\Delta}_{k}\right)$ is nonempty is the one related to $\tilde{U}_{k}$ and $\tilde{X}_{k}$.

(3): Consider again the state sequence $\tilde{X}_{k}\left(x_{k}\right)$. Since $\tilde{X}_{k}\left(x_{k}\right) \in X_{T}^{N}$, inequality (10c) from Assumption 1 holds for all elements in the sequence $\tilde{X}_{k}$, yielding

$$
\begin{aligned}
& F\left(\tilde{x}_{1 \mid k}\right)-F\left(\tilde{x}_{0 \mid k}\right)+L\left(\tilde{x}_{0 \mid k}, h\left(\tilde{x}_{0 \mid k}\right)\right) \leq 0 \\
& F\left(\tilde{x}_{2 \mid k}\right)-F\left(\tilde{x}_{1 \mid k}\right)+L\left(\tilde{x}_{1 \mid k}, h\left(\tilde{x}_{1 \mid k}\right)\right) \leq 0 \\
& \cdots \\
& F\left(\tilde{x}_{N \mid k}\right)-F\left(\tilde{x}_{N-1 \mid k}\right)+L\left(\tilde{x}_{N-1 \mid k}, h\left(\tilde{x}_{N-1 \mid k}\right)\right) \leq 0
\end{aligned}
$$

From these inequalities, by optimality and by Remark 2 it follows that

$$
V_{\mathrm{MPC}}\left(x_{k}\right) \leq J\left(x_{k}, \tilde{U}_{k}\right) \leq F\left(x_{k}\right) \leq \alpha_{2}\left(\left\|x_{k}\right\|\right) \forall x_{k} \in \tilde{\mathcal{N}}
$$

where $\widetilde{\mathcal{N}}=\mathcal{N} \cap \mathbb{X}_{T}$. Again using optimality we have

$$
\begin{aligned}
V_{\mathrm{MPC}}\left(x_{k+1}\right)-V_{\mathrm{MPC}}\left(x_{k}\right) & =J\left(x_{k+1}, U_{k+1}^{*}\right)-J\left(x_{k}, U_{k}^{*}\right) \\
& \leq J\left(x_{k+1}, U_{k+1}^{1}\right)-J\left(x_{k}, U_{k}^{*}\right) \\
& =-L\left(x_{k}, u_{\mathrm{MPC}}\left(x_{k}\right)\right)+F\left(x_{N \mid k+1}\right) \\
& -F\left(x_{N \mid k}^{*}\right)+L\left(x_{N \mid k}^{*}, h\left(x_{N \mid k}^{*}\right)\right)
\end{aligned}
$$

Then as $x_{N \mid k}^{*} \in \mathbb{X}_{T}$ and by condition (10c) in Assumption 1 it holds that

$$
\begin{array}{r}
V_{\mathrm{MPC}}\left(g\left(x_{k}, u_{\mathrm{MPC}}\left(x_{k}\right)\right)\right)-V_{\mathrm{MPC}}\left(x_{k}\right) \leq \\
-L\left(x_{k}, u_{\mathrm{MPC}}\left(x_{k}\right)\right) \leq-\alpha_{1}\left(\left\|x_{k}\right\|\right) \\
\forall x_{k} \in \mathbb{X}_{f}(N)
\end{array}
$$

Since $\mathbb{X}$ is compact and $\mathbb{X}_{f} \subset \mathbb{X}$, then according to 1 ), $\mathbb{X}_{f}(N)$ is positively invariant. Let $x_{k}$ be a state reached with the closed loop system (2) and (9) from initial state $x_{0}$. Chose any $\eta>0$ such that the ball $\mathcal{B}_{\eta} \triangleq\left\{x \in \mathbb{R}^{n} \mid\|x\| \leq \eta\right\}$ satisfies $\mathcal{B}_{\eta} \subset \widetilde{\mathcal{N}}$. It is possible to chose any $0<\epsilon \leq \eta$ a $\sigma \in(0, \epsilon)$ such that $\alpha(\sigma)<\alpha(\epsilon)$. For any $x_{0} \in \mathcal{B}_{\sigma} \subset$ $\mathbb{X}_{f}(N)$, due to positive invariance of $\mathbb{X}_{f}(N)$, it follows that

$$
\begin{aligned}
\cdots & \leq V_{\mathrm{MPC}}\left(x_{k+1}\right) \leq V_{\mathrm{MPC}}\left(x_{k}\right) \leq \cdots \leq V_{\mathrm{MPC}}\left(x_{0}\right) \\
& \leq \alpha_{2}\left(\left\|x_{0}\right\|\right) \leq \alpha_{2}(\sigma) \leq \alpha_{1}(\epsilon)
\end{aligned}
$$

Since we have $V_{\mathrm{MPC}}(x) \geq \alpha_{1}(\epsilon)$ for all $x \in \mathbb{X}_{f}(N) \backslash \mathcal{B}_{\epsilon}$ it follows that $x_{k} \in \mathcal{B}_{\epsilon}$ for all $k \in Z_{+}$.

When the cost function (6) is defined for instance by $F\left(x_{k}\right)=\left\|P x_{k}\right\|_{p}$, and $L\left(x_{k}, u_{k}\right)=\left\|Q x_{k}\right\|_{p}+\left\|R u_{k}\right\|_{p}$, $p=1, \infty$, or $F\left(x_{k}\right)=\left\|P x_{k}\right\|_{2}^{2}$, and $L\left(x_{k}, u_{k}\right)=\left\|Q x_{k}\right\|_{2}^{2}+$ $\left\|R u_{k}\right\|_{2}^{2}$, the computation of the weight matrix $P$ and the state-feedback gain $K$ to define a local control law $h\left(x_{k}\right)$ that fulfill Assumption 1 is described in [14]. These computations are done offline based on different methods and algorithms discussed in the mentioned reference and in the references therein.

\section{Practical issues}

An important practical problem in the proposed method is to find $\bar{\Delta}_{k}$ so that $\mathcal{U}_{N}\left(x_{k}, \bar{\Delta}_{k}\right)$ is nonempty, so that the MIP including mode sequence constraints has a solution. When states are affected by measurement noise and modeling disturbances are present, the assumption that $x_{0 \mid k}=x_{1 \mid k-1}$ will not hold and the shifted sequence from the previous sample will not necessary be feasible. Finding $\bar{\Delta}_{k}$ by using (11) is therefore not an option in the presence of nondeterminism. 
The simplest way to find $\bar{\Delta}_{k}$ is to simulate in openloop the hybrid dynamics (2) (e.g. through its piecewise affine equivalent form [15], [16] or directly form a HYSDEL simulator [11], [16]) from initial condition $x_{k}$ under the control input $U_{k}^{1}$ defined by (12). If all constraints are respected, $U_{k}^{1}$ is a feasible solution and $\bar{\Delta}_{k}$ can be directly defined as the simulated $\delta$-trajectory. If the measured state is close to the predicted state, it is reasonable to believe that this sequence provides at least a good initial guess close the the optimum.

If the open-loop simulation fails because some constraints are violated, choosing $\bar{\Delta}_{k}$ amounts to finding a feasible trajectory for the problem without mode sequence constraints from the new initial state. This in turn is a MIP feasibility problem. The reduction in time that can be achieved with the presented methodology then depends on the complexity of feasibility problem compared to the optimization problem, something that is difficult to analyze a priori. This is a restriction to the presented method but if constraints related to safety or high risk are present in $\mathbb{X}$, and feasibility can not be assured within a pre-specified time-frame, neither the presented method nor other hybrid MPC strategies that depend on a MIP to find a feasible solution would be applicable in practice. Indeed, in all practical applications of MPC one resorts to "softening" all the constraints that involve state variables to prevent infeasibility issues (that is, all constraints but input saturation).

\section{A. Relaxing the stability requirement}

Lack of stability guarantees has not been an obstacle for the practical application of MPC in the past. Before any stability proofs had been presented for MPC controllers they had been extensively applied on a variety of control problems in industry. Moreover, stability proves usually hold under the assumption that the model and the actual process coincide, which is hardly true in practice.

In this section some guidelines are given on how to find the sequence $\bar{\Delta}_{k}$ when the requirement for stability is relaxed.

If $\mathbb{X}=\mathbb{X}_{T}=\mathbb{R}^{n}$ (no state constraints) and $\mathbb{U}$ is a box in $\mathbb{R}^{m}$ corresponding to actuator saturation constraints, then $U_{k}^{1} \triangleq\left(u_{1 \mid k-1}^{*}, \ldots, u_{N-1 \mid k-1}^{*}, \operatorname{sat}\left(h\left(x_{N \mid k-1}^{*}\right)\right)\right)$ can be used to generate $\bar{\Delta}_{k}$ by open-loop simulation.

On the other hand, state constraints are generally related to either physical constraints of the model such as conservation equations and physical limitations of the process, or to control objectives. The way infeasibility in the optimization problem is dealt with, or constraint management, is an important issue in constrained predictive control, see [12].

As mentioned above, a common approach to deal with infeasibility is to change constraints from "hard" to "soft", that is, add terms containing slack variables of the constraints to the cost function. If the constraints thus changed represent physical characteristics, the resulting control signal might be of little use as the model from which the control signal is obtained might not fulfill basic physical laws. If the constraints are related to safety considerations, the resulting control signal might not be applicable either.
Constraint management is equally important in the presented scheme as a straightforward way to obtain an initial feasible solution is to change any unfulfilled constraints in $\mathbb{X}$, when $U_{k}^{1}$ is used in open-loop simulation, into soft constraints. As mentioned previously, this approach is only appropriate if the constraints thus relaxed do not represent physical or safety characteristics of the system.

In some situations, physics or heuristical knowledge of the system can often be used to find a feasible solution that fulfills the physical constraints of the system. For example, in steady state, all integer variables have fixed values which could be used to define the sequence $\bar{\Delta}_{k}$.

\section{EXAMPLE}

In order to show the effectiveness of the discussed technique, let us consider the simple system proposed in [14], which is described in PWA form as

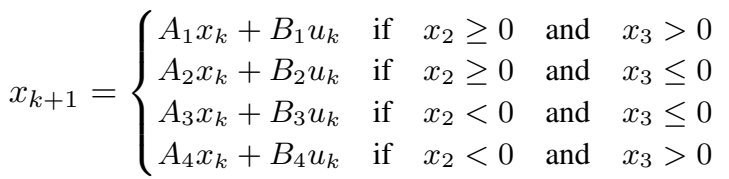

subject to the constraints $x_{k}=[-5,5]^{3}$ and $u_{k} \in[-1,1]$. Due to limitations on space, only results related to the use of $M$ constraints are presented.

Using the equivalence between PWA and MLD forms [15], the corresponding HYSDEL code [11] was written defining 6 binary and 12 continuous auxiliary variables. As in [2], an MPC control law (9) was computed by setting the cost function using $\infty$-norms for a horizon $N=4$ and weight matrices $Q=0.02 I_{3}, R=0.01$. The numerical values for the system matrices as well as the terminal state weight $P$ and state feedback gains $K_{i}$ for the local control law $h(x)=$ $K_{i} x_{k}$ can be found in appendix. As all states are assumed to be measurable and there are no disturbances, the sequence $\bar{\Delta}_{k}$ was found by using the local control law given by the state feedback gains and using (11).

The closed-loop system was simulated for 15 samples using the HyBRID TOOLBOX for MATLAB [16]. and the MIP solver CPLEX 9.1 [17] from the initial state $x_{0}=$ $\left[\begin{array}{lll}-3 & 1 & -5\end{array}\right]^{T}$. At the end of the simulation scenario, the trajectory always converges to the terminal set as defined in [14].

Figure 1 shows the average optimization time at each sample for distinct values of $M$. Also shown is the average time when no mode sequence constraints are present. What is presented is the the average of the optimization time when the problem at each sample is solved 100 times. This is due to the fact that CPLEX contains certain random initial settings, causing a difference in calculation time even though the MIP problem to be solved is the same.

The main conclusion that can be drawn from Figure 1 are the following:

- There is a considerable reduction in optimization time for $M=0,1,2$ compared to the other cases. Considering sample 3 , while the other cases have a calculation time of at least 0.06 seconds, the maximum calculation 


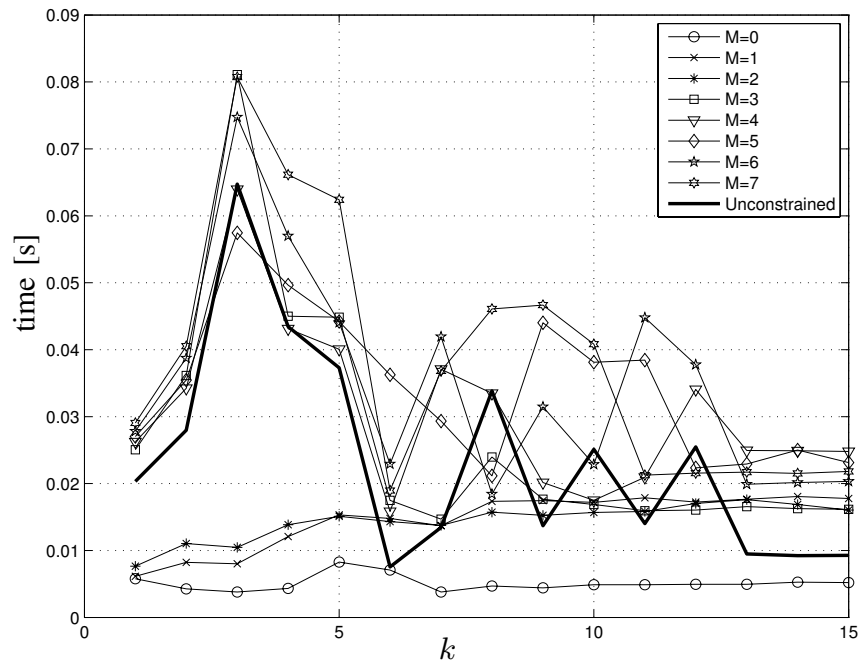

Fig. 1. Average optimization time as a function of $M$ at each sample. Also shown is the average simulation time in the unconstrained case (bold line).

time for the cases $M=0,1,2$ never goes above 0.02 seconds. This means that the modes sequence constraints for $M=0,1,2$ reduce the worst case calculation time to one third of the worst case calculation time for other values of $M$ and the unconstrained case.

- There is a considerable sample to sample variance in optimization time for the values of $M$ distinct from $0,1,2$. On the other hand there is correlation in the optimization time, indicating that the problem complexity changes as a function of the time index $k$.

Figure 3 shows the sum of the calculation time for the first 7 samples. The reason why 7 samples were chosen can be seen in Fig. 2. There the norm of the state vector is shown as a function of $k$, demonstrating the convergence of the state to zero. At time 7 all states had a norm smaller than $0.1 \%$ of the initial norm. Convergence after that point was much smaller and seemed affected by numerical issues. Note that there is very small difference between the trajectories at each time. Only at time $k=4$ there is a noticeable difference.

Figure 3 shows that there is an important jump in optimization time when $M$ changes from 2 to 3 . For $M$ smaller than 3 an important reduction in optimization time is observed. For $M$ large or equal to 3, adding mode sequence constraints does not improve optimization time but rather deteriorates it.

To obtain an idea of the level of suboptimality in terms of the cost function optimized, Figure 4 shows the sum of the cost functions over the scenario for each value of $M$, normalized with the sum of the cost functions when no mode sequence constraints are present, that is:

$$
\mathcal{S}(M)=\sum_{k=1}^{15} J_{k}(M) / \sum_{k=1}^{17} J_{k} \times 100 \%
$$

where $J_{k}(M)$ is the cost function for a specific $M$, and $J_{k}$ is the cost function without mode sequence constraints.

It can be seen that $\mathcal{S}(M)$ increases when $M$ goes to zero.

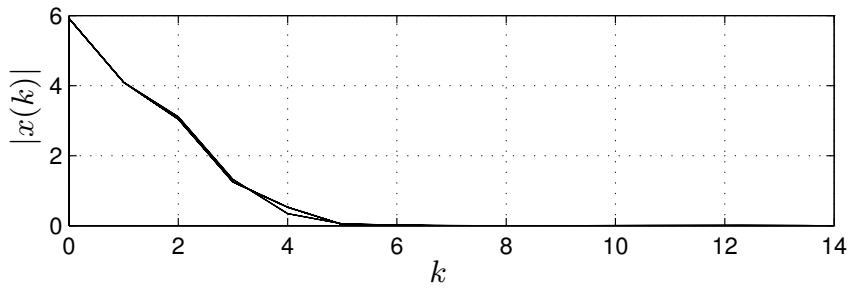

Fig. 2. 2-norm of state vector as a function of time $k$.

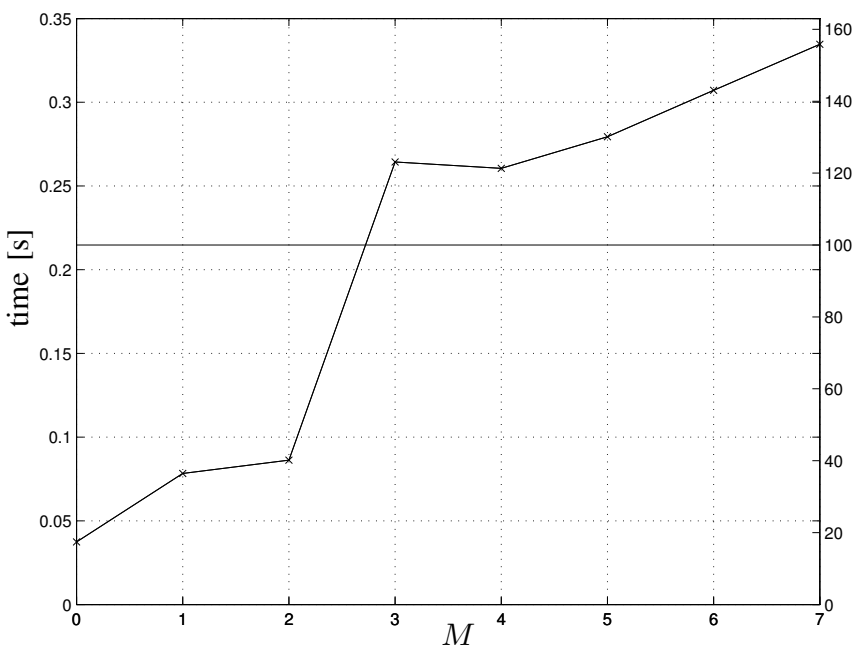

Fig. 3. Average simulation time as a function of $M$. The horizontal line is the average calculation time without mode sequence constraints. On the right axis the simulation time as a function of $M$ is shown as a percentage of calculation time without mode sequence constraints.

When $M=0$, the cost is around $6 \%$ higher than the cost without mode sequence constraints. It is also seen that for $M=3$ the proposed scheme is not suboptimal as $\mathcal{S}(M)=$ $100 \%$. On the other hand, as shown before, no reduction in optimization time is observed for $M \leq 3$. The reduction in optimization time results therefore in an increase in the cost function.

\section{CONCLUSIONS AND FUTURE WORK}

A suboptimal model predictive control scheme for discrete-time hybrid systems has been presented where optimality is sacrificed for a reduction of computation time. Stability of the scheme has been proven when states are

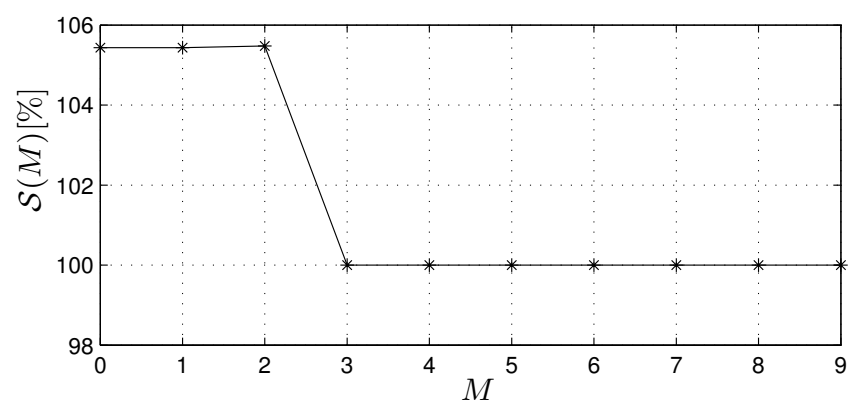

Fig. 4. Suboptimality level $\mathcal{S}(M)$. 
measured and no disturbances are present. It has been shown that by using the proposed scheme, calculation time can be reduced consistently as a function of the maximum number $M$ of allowed switches from a reference switching sequence.

Some important issues not addressed here due to space limitations are for example how to select $M$ or $M_{i}$ in a systematic way. As discussed in Section IV, the applicability of the method depends on availability of the sequence $\bar{\Delta}_{k}$. Systematic ways to find $\bar{\Delta}_{k}$ without solving a MIP feasibility problem but taking into account physical knowledge of state constraints can also be investigated. Furthermore, the method will be applied to large scale systems where calculation time is very difficult to predict.

\section{ACKNOWLEDGMENTS}

This work has been supported by the CICYT projects with reference nr. DPI2005-04722, DPI2002-03500 and the Ramon y Cajal Program, both of the Spanish Science and Technology Ministry. Support has also been received by the DGR of Generalitat de Catalunya (SAC group Ref. 2005/SGR/00537), by the European Commission under the HYCON Network of Excellence, contract number FP6-IST511368, and by the Italian Ministry for Education, University and Research (MIUR) under project "Advanced control methodologies for hybrid dynamical systems" (PRIN'2005).

\section{APPENDIX}

The numerical values for the system matrices are:

$$
\begin{array}{lll}
A_{1}=\left[\begin{array}{ccc}
-0.2523 & 0.4856 & 0.4856 \\
0.5290 & -0.2616 & -0.2616 \\
-0.4415 & -0.2713 & -0.2713
\end{array}\right], \quad B_{1}=\left[\begin{array}{c}
0.5656 \\
0.5460 \\
0.9389
\end{array}\right], \\
A_{2}=\left[\begin{array}{ccc}
0.0647 & 0.1729 & -0.6542 \\
-0.3131 & -0.6691 & -0.6516 \\
-0.3085 & 0.0613 & 0.0099
\end{array}\right], & B_{2}=\left[\begin{array}{c}
0.6543 \\
0.5266 \\
-0.0558
\end{array}\right], \\
A_{3}=\left[\begin{array}{ccc}
0.6402 & -0.5409 & -0.5629 \\
-0.6693 & -0.6874 & 0.1748 \\
-0.2812 & 0.4898 & -0.3526
\end{array}\right], & B_{3}=\left[\begin{array}{c}
0.7580 \\
-0.8050 \\
-0.4059
\end{array}\right], \\
A_{4}=\left[\begin{array}{ccc}
-0.3501 & 0.2590 & 0.6695 \\
-0.4808 & 0.1905 & 0.3865 \\
-0.1217 & -0.2631 & -0.0013
\end{array}\right], & B_{4}=\left[\begin{array}{c}
0.6961 \\
-0.7619 \\
-0.2590
\end{array}\right] .
\end{array}
$$

The weight matrix for the terminal state and gains for the local state-feedback stabilizing controller are:

$$
\begin{aligned}
P & =\left[\begin{array}{ccc}
0.7029 & 3.8486 & 1.1501 \\
4.1796 & 0.5642 & 1.6656 \\
-1.4275 & 1.5026 & 5.3197 \\
-1.3717 & 2.5343 & -1.5468
\end{array}\right], \\
K_{1} & =\left[\begin{array}{lll}
0.4699 & 0.1750 & 0.1591
\end{array}\right], \\
K_{2} & =\left[\begin{array}{lll}
0.4039 & 0.4239 & 1.1529
\end{array}\right], \\
K_{3} & =\left[\begin{array}{lll}
-0.7742 & -0.1436 & -0.1603
\end{array}\right], \\
K_{4} & =\left[\begin{array}{lll}
-0.0800 & -0.0405 & -0.2867
\end{array}\right] .
\end{aligned}
$$

They were taken from the computations described in [14] for the case of a cost function based on infinity norms.

\section{REFERENCES}

[1] A. Bemporad and M. Morari, "Control of systems integrating logic, dynamics, and constraints," Automatica, vol. 35, no. 3, pp. 407-427, 1999.

[2] M. Lazar, W. Heemels, S. Weiland, and A. Bemporad, "Stability of hybrid model predictive control," IEEE Transactions of Automatic Control, vol. 51, no. 11, pp. 1813 - 1818, 2006.

[3] D. Mayne, J. Rawlings, C. Rao, and P. Scokaert, "Constrained model predictive control: Stability and optimality," Automatica, vol. 36, pp. 789-814, 2000.

[4] C. Ocampo-Martínez, A. Bemporad, A. Ingimundarson, and V. Puig, "On hybrid model predictive control of sewer networks," in Identification \& Control: The gap between theory and practice, R. SánchezPeña, V. Puig, and J. Quevedo, Eds. Springer-Verlag, 2007.

[5] C. G. Cassandras and R. Mookherjee, "Receding horizon control for a class of hybrid systems with event uncertainties," in ACC, 2003.

[6] B. De Schutter and T. van den Boom, "Model predictive control for max-plus-linear discrete event systems," Automatica, vol. 37, no. 7, pp. 1049-1056, July 2001.

[7] M. S. Branicky, V. S. Borkar, and S. K. Mitter, "A unified framework for hybrid control: Model and optimal control theory," IEEE Transactions on Automatic Control, vol. 43, no. 1, pp. 31-45, 1998.

[8] X. Xu and P. J.Antsaklis, "Optimal control of switched systems based on parameterization of the switching instants," IEEE Trans. Automatic Control, vol. 49, pp. 2-16, January 2004.

[9] M. Shaikh and P. Caines, "On the hybrid optimal control problem: Theory and algorithms," IEEE Trans. Automatic Control, 2007, in press.

[10] C. Seatzu, D. Corona, A. Giua, and A. Bemporad, "Optimal control of continuous-time switched affine systems," IEEE Trans. Automatic Control, vol. 51, no. 5, pp. 726-741, 2006.

[11] F. Torrisi and A. Bemporad, "Hysdel - A tool for generating computational hybrid models for analysis and synthesis problems," IEEE Trans. Contr. Syst. Technol., vol. 12, no. 2, pp. 235-249, 2004.

[12] J. Maciejowski, Predictive Control with Constraints. Great Britain: Prentice Hall, 2002.

[13] E. Camacho and C. Bordons, Model Predictive Control, 2nd ed. Springer-Verlag, London, 2004.

[14] M. Lazar, "Model predictive control of hybrid systems: Stability and robustness," Ph.D. dissertation, Technische Universiteit Eindhoven, 2006.

[15] W. Heemels, B. De Schutter, and A. Bemporad, "Equivalence of hybrid dynamical models," Automatica, vol. 37, pp. 1085-1091, 2001.

[16] A. Bemporad, Hybrid Toolbox - User's Guide, April 2006. [Online]. Available: http://www.dii.unisi.it/hybrid/toolbox

[17] I. S. A., ILOG CPLEX 9.1 User's Manual, 2003. 